Article

\title{
Experimental Validation of Optimal Parameter and Uncertainty Estimation for Structural Systems Using a Shuffled Complex Evolution Metropolis Algorithm
}

\author{
Hesheng Tang ${ }^{1,2}$, Xueyuan Guo ${ }^{1} \mathbb{( D}$, Liyu Xie $^{1, * \mathbb{C}}$ and Songtao Xue ${ }^{1}$ \\ 1 Department of Disaster Mitigation for Structures, Tongji University, Shanghai 200092, China; \\ thstj@tongji.edu.cn (H.T.); guoxueyuan@tongji.edu.cn (X.G.); xue@tongji.edu.cn (S.X.) \\ 2 State Key Laboratory of Disaster Reduction in Civil Engineering, Tongji University, Shanghai 200092, China \\ * Correspondence: liyuxie@tongji.edu.cn; Tel.: +86-21-6598-2390
}

Received: 6 September 2019; Accepted: 11 November 2019; Published: 18 November 2019

\begin{abstract}
The uncertainty in parameter estimation arises from structural systems' input and output measured errors and from structural model errors. An experimental verification of the shuffled complex evolution metropolis algorithm (SCEM-UA) for identifying the optimal parameters of structural systems and estimating their uncertainty is presented. First, the estimation framework is theoretically developed. The SCEM-UA algorithm is employed to search through feasible parameters' space and to infer the posterior distribution of the parameters automatically. The resulting posterior parameter distribution then provides the most likely estimation of parameter sets that produces the best model performance. The algorithm is subsequently validated through both numerical simulation and shaking table experiment for estimating the parameters of structural systems considering the uncertainty of available information. Finally, the proposed algorithm is extended to identify the uncertain physical parameters of a nonlinear structural system with a particle mass tuned damper (PTMD). The results demonstrate that the proposed algorithm can effectively estimate parameters with uncertainty for nonlinear structural systems, and it has a stronger anti-noise capability. Notably, the SCEM-UA method not only shows better global optimization capability compared with other heuristic optimization methods, but it also has the ability to simultaneously estimate the uncertainties associated with the posterior distributions of the structural parameters within a single optimization run.
\end{abstract}

Keywords: parameter identification; uncertainty estimation; Markov chain Monte Carlo; shuffled complex evolution metropolis algorithm; optimization algorithm

\section{Introduction}

Nowadays, parameter estimation with high accuracy and practicality is essential in civil engineering when assessing the performance of structural systems; thus, a contractor, engineer, or assessor will use it when evaluating such issues as reliability predictions, structural control, and structural health monitoring. Parameter estimation takes aim at searching for the model parameter values that has the best fit to measurement data. Therefore, it provides a reliable model to describe the current state of the structure [1]. Structural parameter identification is based on least squares or maximum likelihood criterion generally [2,3], which requires solving a nonlinear and nonconvex optimization problem. The error surface in this estimation problem is a hypersurface in the multidimensional parameter space, thereby the hypersurface potential is highly multimodal possessing local optimum. This may lead to the search algorithms converging to local optimum or being trapped in suboptimal regions. To surmount these problems, global optimization algorithms are recommended [4]. Recently, global heuristic computational intelligence methods have been found to 
be powerful when local methods have not proven effective. Global heuristic optimization methods are an active field of research with more models and strategies proposed every year. Some examples are genetic algorithms (GA) [5,6], differential evolution [7,8], particle swarm optimization (PSO) [9,10], the Big Bang-Big Crunch (BB-BC) algorithm [11], evolution strategy (ES) [12], simulated annealing [13], the Shuffled Complex Evolution (SCE-UA), and some variations of the SCE-UA model [14,15].

While some global optimization methods focus on finding the best set of parameters, a realistic assessment of parametric uncertainty in structural systems has not been given enough attention. The identification of parameters involves uncertainties that are caused by mathematical model simplification, errors in the measured data, and insufficient bandwidth. Consequently, finding a single optimal set in the parameter space is generally impossible. The identified parameter set should be represented with a confidence range. Classical deterministic approaches quite often involve minimization of the least-squares norm, which compares the system measured response to model predictions with some kind of regularization. However, neither errors in the measured data nor uncertainties in model predictions are rigorously accounted for [16]. Although these global optimization methods are extensively used and are robust and efficient for parameter estimation, finding out a unique "optimal" parameter set remains a huge challenge, which has a significantly better performance compared with other feasible parameter sets [17].

Realistic quantification of parameter uncertainty greatly influences structural analysis results and determines the final decision of structural design, assessment, and control strategies. Recently proposed methods for the estimating of parametric uncertainty in structural models, including the Markov chain Monte Carlo (MCMC) algorithms [18,19] and bootstrap techniques [20-22], have been successfully applied to update structural models and estimate their parameter uncertainties. MCMC algorithms have received increasing attentions for sampling from a posterior probability distribution of structural parameters of interests [23-25]. However, MCMC algorithms require a prior probability sampling distribution determining explorative capabilities and convergence rate [17]. For complex structures, it is impossible to rigorously incorporate any relevant information, including model and measurement uncertainties. Prior knowledge about the high probability region located in the parameter space is usually insufficient. Therefore, the sampling driven by transitions of Markov chain is used to adjust the proposal distribution to the posterior target distribution, which improves the efficiency of MCMC algorithms. Vrugt et al. developed an adaptive MCMC algorithm, i.e., the Shuffled Complex Evolution Metropolis algorithm (SCEM-UA) [17]. The SCEM-UA merges the superiorities of controlled random searches [26], competitive evolution [27], complex shuffling [14], and Metropolis algorithm [28]. Vrugt et al. claimed that the SCEM-UA algorithm has wide applicability for parameter optimization and uncertainty assessment, and can calculate the prediction interval better than the SCE-UA algorithm due to the stochastic character of the Metropolis algorithm. The SCEM-UA algorithm has been proven to possess effectiveness for calibrating the groundwater model and the conceptual rainfall-runoff model already [17,29-31]. Previous studies have indicated that the SCEM-UA algorithm possesses adaptive capabilities, and it can significantly alleviate computational burden by reducing the number of simulations during the evolution of the parameter posterior distribution [17,32]. The SCEM-UA method has been applied many times for realistic assessments of parameter and model prediction uncertainties of hydrologic models; however, to the best of the authors' knowledge, the algorithm has not been applied to parameter optimization and uncertainty assessments in civil engineering.

In this work, a parameter identification method is employed to assess uncertainty in the parameter estimates of structural models using the SCEM-UA algorithm. The outline of the paper is as follows. In Section 2, the framework of parameter identification for a structural system is developed. Besides, the SCEM-UA algorithm is briefly discussed. To verify the accuracy of the identification method, numerical studies are conducted for the shear models based on pseudo-experimental data under various noise conditions in Section 3. Then in Section 4, the proposed parameter estimation algorithm is validated through experiments on scale-models of both linear and nonlinear structural systems, 
where the physical parameters of the shear frame and the additional damping system are identified. Finally, Section 5 is devoted to some conclusions.

\section{Methodology}

\subsection{Bayesian Framework for Parameter Estimation of Structural System}

It is assumed that the numerical model of the structural system is predefined and fixed. The functional form, here the multiple-degree-of-freedom (MDOF) structural model $\eta$, is used to relate system response with unknown quantities and can be written as

$$
\hat{\mathbf{y}}=\eta(\xi \mid \theta)+e,
$$

where $\hat{\mathbf{y}}=\left(\mathbf{y}_{1}, \mathbf{y}_{2}, \cdots \mathbf{y}_{\mathbf{n y}}\right)^{T}$ is the vector of model outputs, such as displacement, velocity or acceleration; $\boldsymbol{\theta}=\left(\theta_{1}, \theta_{2}, \cdots, \theta_{n}\right)$ is a vector of $n$ structural model parameters to be estimated, and $\xi$ is the input values to the structural model. Finally, e represents modeling residuals, which consists of various sources of prediction errors. However, it has been observed that many parameter sets close to the global optimum within the feasible parameter space show similar performances of reproducing the observed data $[17,29]$.

In Bayesian inference, the uncertainties of the random parameter variables $\theta$ before any data are collected, are described by its probability density functions (pdf), denoted as prior $p(\theta)$. The posterior pdf, $p(\theta \mid \mathbf{y})$, confirms the parameters functions given as the observed measurements $\mathbf{y}$, and is proportional to the product of the prior pdf and the likelihood function. The posterior distribution is the final goal of the statistical inversion approach, i.e., the solution of the inverse problem. Notably, once a prior probability distribution has been defined, it is possible to weigh its influence over posterior density outcomes based on the initial state of knowledge about the parameters. If the prior information is insufficient or not reliable, a non-informative prior density should be employed (for instance, a uniform distribution); consequently, the information contained in the data has a predominant effect on the inverse solution in the Bayesian inference scheme.

Typically, for complex structural models, the joint posterior pdf $p(\theta \mid \mathrm{y})$ is multidimensional, complex, and usually unknown, with strong interdependences of nonlinear parameter. $p(\theta \mid \mathrm{y})$ is thereby difficult for direct sampling or analytical integration. The prior information is usually defined by the feasible parameter space, $\theta \in \Theta \in R^{n}$, consisting of lower and upper bounds on uniform distribution (non-informative) of each parameter. The uniform assignment is chosen mostly for its simplicity to encode a lot of initial ignorance about the parameters. In this research, the authors employ the SCEM-UA algorithm to tune a sequence of parameter sets $\theta=\left(\theta_{1}, \theta_{2}, \cdots, \theta_{n}\right)$ to the target posterior distribution.

\subsection{Parameter Estimation with the SCEM-UA Algorithm}

This algorithm assumes an inherent uncertainty in parameter identification, partly due to measurement error, model output error, or the fact that the model does not perfectly represent the model. The SCEM-UA algorithm coordinates with a Bayesian scheme where parameters are represented as probabilities instead of as a single optimal set of values to derive the posterior pdf of the structural system parameters.

The SCEM-UA algorithm starts with a population of $s$ parameter sets. The parameter sets randomly distribute within the feasible parameter space, which is defined by the joint prior parameter distributions $p(\theta)$ initially. The posterior density $p(\theta \mid \mathbf{y})$ is computed by Bayesian inference scheme for each parameter set. A total of $s$ samples from the parameter space $\theta$ are stored in order of decreasing $p(\theta \mid \mathbf{y})$. The sample points are partitioned into $q$ complexes with the expectation that $q<<s$. Partitioning is done by striping the total population across the complexes. More precisely, complex $c$ contains all samples numbered $i$ such that $i \bmod q=c$. After the samples have been divided into complexes, each complex is evolved independently employing the sequence evolution metropolis (SEM) algorithm. 
This SEM algorithm operates in each parallel sequence to generate new candidate points from an adaptive proposal distribution based on the information derived from the $\mathrm{m}$ samples. After the SEM algorithm is completed, all the updated complexes are combined and stored in order of decreasing posterior density. The evolution and shuffling will stop when the Gelman-Rubin convergence criteria is achieved, which demonstrates convergence to a stationary distribution [33].

The operations including use of complexes during evolution and the shuffling of these complexes conduct an efficient and robust search for the optimal parameter set. The SCEM-UA algorithm collects information of each individual sequence and enhances the survivability of each sequence by globally sharing the information during the evolution process. The flowchart diagram shows the SCEM-UA algorithm implementation scheme as shown in Figure 1. Please refer to Vrugt et al. and Feyen et al. for more information about the algorithm $[17,29]$.

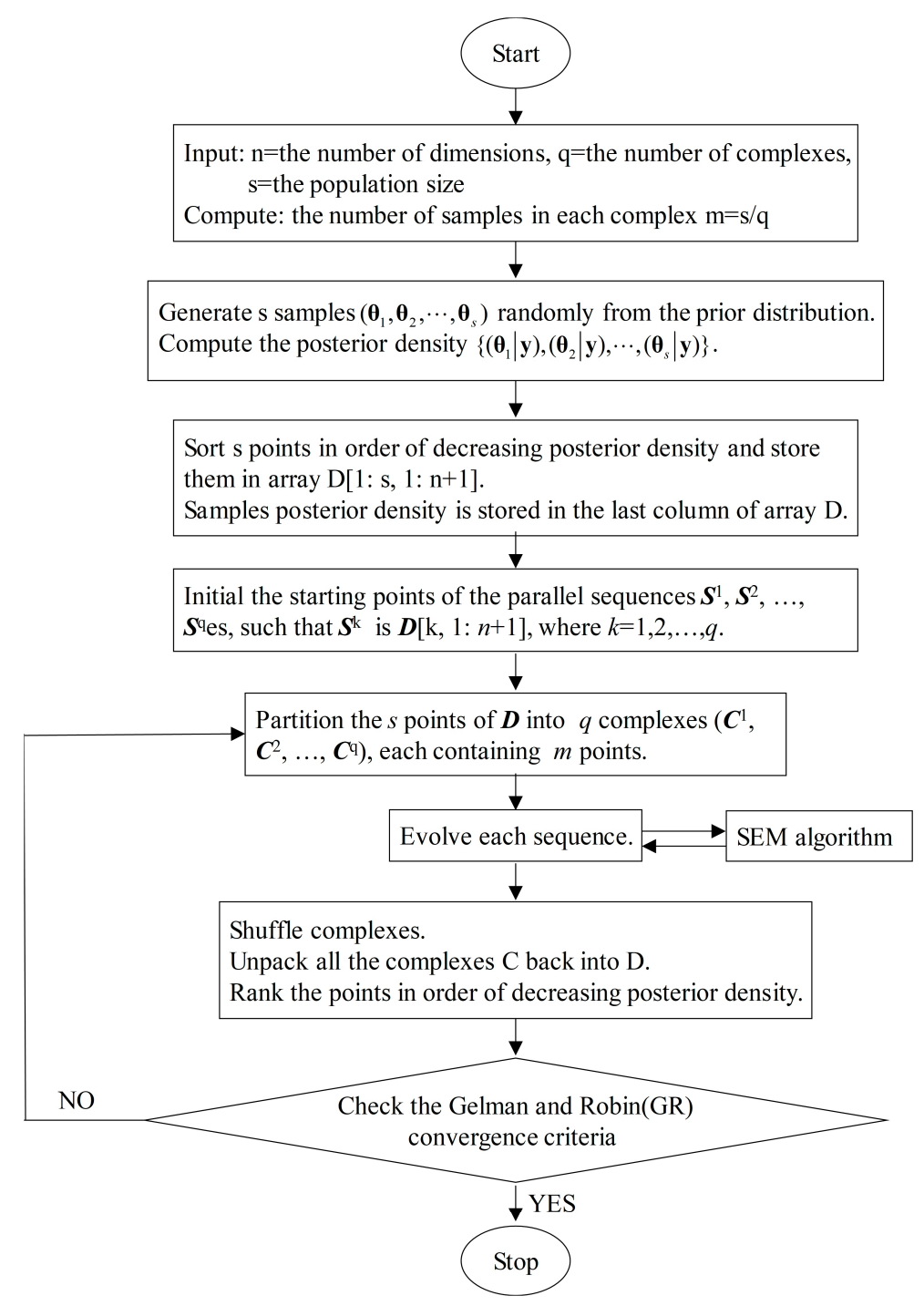

Figure 1. The flowchart of the algorithm.

\section{Numerical Studies}

To investigate the effectiveness of the SCEM-UA algorithm for parameter estimation and uncertainty assessment of structural models, numerical simulations of MDOF structural system models are carried out. The two-dimensional shear frame type structure shown in Figure 2 is analyzed. The dynamic equation of the structure motion can be described as 


$$
\mathbf{M} \ddot{\mathbf{x}}(t)+\mathbf{C} \dot{\mathbf{x}}(t)+\mathbf{K x}(t)=\mathbf{F}(t)
$$

where $\mathbf{M}, \mathbf{C}$, and $\mathbf{K}$ represent the mass, damping and stiffness matrices, respectively. $\mathbf{F}$ is the input force vector, and $\mathbf{x}$ is the displacement vector. The mass of each floor is concentrated and the damping is assumed as the Rayleigh damping matrix $\mathbf{C}$. The damping ratio, $\zeta_{\mathrm{r}}$, of the first two modes of vibration. Therefore, the n-DOF structure system can be described by the parameter set $\theta=\left(m_{1}, m_{2}, \ldots, m_{n}, k_{1}\right.$, $\left.k_{2}, \ldots, k_{n}, \zeta_{1}, \zeta_{2}\right)$.
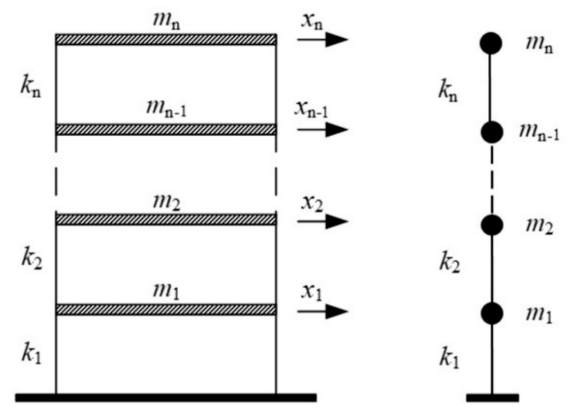

Figure 2. n-DOF structure model.

The structure is excited by known forces, $\mathbf{F}(t)$ and the acceleration response of the structure, $\ddot{\mathbf{x}}(t)$, is measured at some given point. Let $\hat{\mathbf{x}}_{j}\left(t_{i}\right)$ also for $j=1,2, \ldots, n y$, and $i=1,2, \ldots, \mathrm{T}$ denotes the value of the $j$ th response of the identified system at the $i$ th time step. All available output records can be written as

$$
\hat{\mathbf{y}}=\left(\hat{\mathbf{x}}_{1}(t), \hat{\mathbf{x}}_{2}(t), \ldots \hat{\mathbf{x}}_{\mathrm{ny}}(t)\right)_{t=t_{1}, \ldots . t_{\mathrm{T}}}^{T} .
$$

First, the authors consider the parameter estimation for an 8-DOF structural model and the structural properties are shown in Table 1 with no prior knowledge about stiffness and damping. The parameters of stiffness and damping are all constant over time and the structural response to a given excitation is carried out by fourth-order Runge-Kutta method. The structure is excited by the Niigata earthquake (Japan, 2000). This paper elucidates the uncertainty assessment for parameter estimations and how their limited available observations with error are handled. The input and output (I/O) data are polluted by Gaussian, zero mean, and white noise. Root mean square (RMS) value of the noise is a certain percentage of the real time histories. The "full output" indicates that measurement data are available at all floors, whereas, the "partial output" indicates that data are available at Floors $1,3,5$, and 7 .

Table 1. Structure properties of the 8-DOF system.

\begin{tabular}{cc}
\hline Mass (kg) & \\
\hline$m_{1} \sim m_{7}$ & 4.95 \\
$m_{8}$ & 4.51 \\
Stiffness $(\mathrm{kN} / \mathrm{m})$ & \\
$k_{1}$ & $5.53 \times 10^{3}$ \\
$k_{2} \sim k_{8}$ & $2.72 \times 10^{3}$ \\
Damping ratio & \\
$\zeta_{1}$ & $1 \times 10^{-2}$ \\
$\zeta_{2}$ & $3 \times 10^{-2}$ \\
\hline
\end{tabular}

The SCEM-UA algorithm was implemented with $\mathrm{q}=10$ complexes including 30 points in each complex, indicating a population size $s=300$. The prior information consists of lower and upper bounds on each parameter distribution as shown in Table 2; thus, a feasible parameter space can be defined and a uniform prior distribution is imposed on this rectangle. Three scenarios are tested: Case1: 0\% noise + Full Outputs; Case2: 10\% noise + Full Outputs, and Case3: 10\% noise + Partial Outputs. 
Table 2. Bounds of the prior uniform parameter distributions.

\begin{tabular}{ccc}
\hline Parameter & Lower Bound & Upper Bound \\
\hline$k_{1}(\mathrm{kN} / \mathrm{m})$ & $2.765 \times 10^{3}$ & $11.06 \times 10^{3}$ \\
$k_{2} \sim k_{8}(\mathrm{kN} / \mathrm{m})$ & $1.36 \times 10^{3}$ & $5.44 \times 10^{3}$ \\
$\zeta_{1}$ & $5 \times 10^{-1}$ & $2 \times 10^{-2}$ \\
$\zeta_{2}$ & $1.5 \times 10^{-2}$ & $6 \times 10^{-2}$ \\
\hline
\end{tabular}

The $\sqrt{S R}$ defined by Gelman and Rubin [33] was utilized to evaluate the convergence of the MCMC algorithm. When the factor is less than 1.2, which indicates the Markov chain is converged to a stationary distribution, the evolution stops. The evolution of the Gelman-Rubin factor for the parameters $k_{1}-k_{8}, \zeta_{1}$ and $\zeta_{2}$ of the three cases using the SCEM-UA algorithms is shown in Figure 3a-c, respectively. For all parameters, the line plots converge to a stationary distribution after approximately 300 iterations. The evolution of the generated samples is illustrated in Figure $4 \mathrm{a}-\mathrm{c}$, confirming the rapid convergence of parameters $k_{1}, k_{5}$, and $\zeta_{1}$ for the three scenarios. Although not presented in this paper, similar results are obtained for the other parameters.

Figure $5 \mathrm{a}-\mathrm{c}$ presents the marginal posterior probability distributions of the estimation parameters $k_{1}, k_{5}$, and $\zeta_{1}$, respectively, for the three scenarios using 6000 samples generated after convergence. Similar results were obtained for the other parameters. Note that the histograms for all parameters shows a normal distribution centered around the realistic parameter value, which means the posterior mean approximates the realistic parameter value. All parameters in Figure $5 \mathrm{a}-\mathrm{c}$ do not correspond to the respective prior uniform distributions. The prior uniform distributions give equal probability to the entire parameter range, which represent the ignorance about the truth of the hypothesis before we have analyzed the current data. The posterior pdf is then dominated by the likelihood function, and the choice of the prior becomes largely irrelevant. The SCEM-UA method considers tuning the prior probability distribution of parameters towards achieving an appropriate posterior probability distribution, during each evolution. This highlights the ability of the SCEM-UA method to search good model parameter sets without any prior knowledge of the system parameters. The summarizing statistics of the posterior parameter distributions are presented in Table 3 for each scenario.

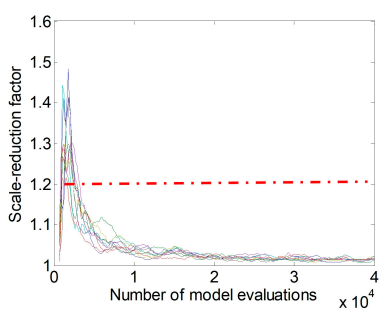

(a)

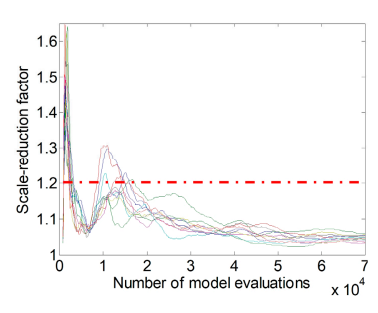

(b)

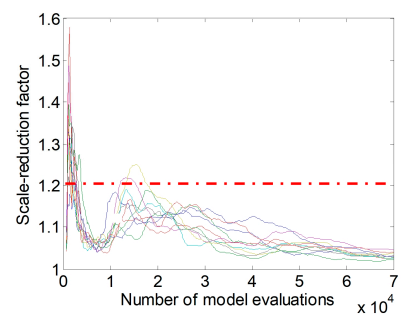

(c)

Figure 3. Evolution of the scale reduction factor for the eight estimation parameters: (a) Case 1: 0\% noise + Full Outputs; (b) Case 2: 10\% noise + Full Outputs; (c) Case 3: 10\% noise + Partial Outputs.
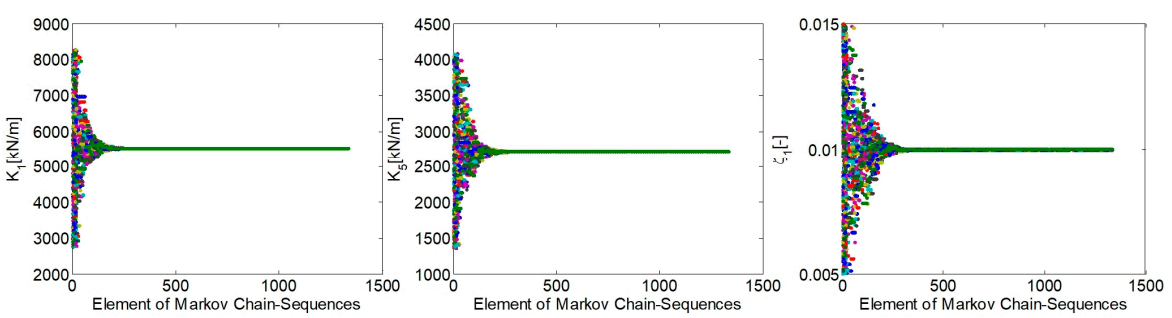

(a)

Figure 4. Cont. 

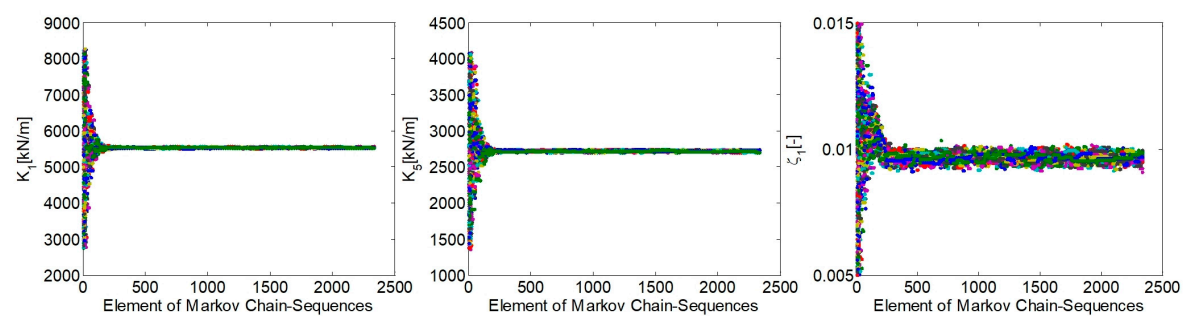

(b)
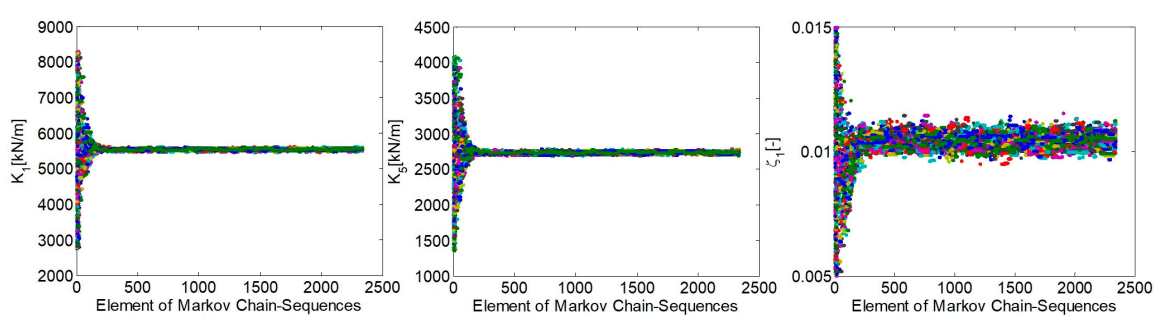

(c)

Figure 4. Generated samples for the three estimation parameters $\left(k_{1}, k_{5}, \zeta_{1}\right)$ : (a) Case 1: $0 \%$ noise + Full Outputs; (b) Case 2: 10\% noise + Full Outputs; (c) Case 3: 10\% noise + Partial Outputs.
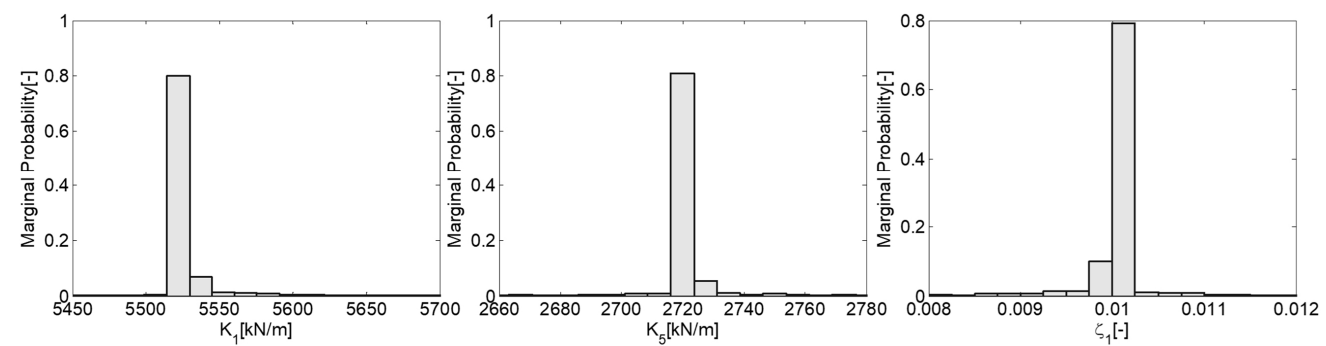

(a)
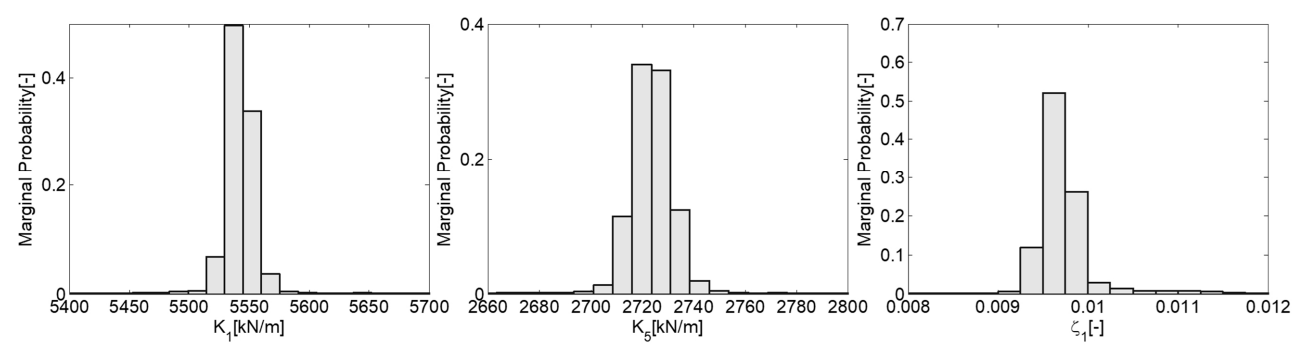

(b)
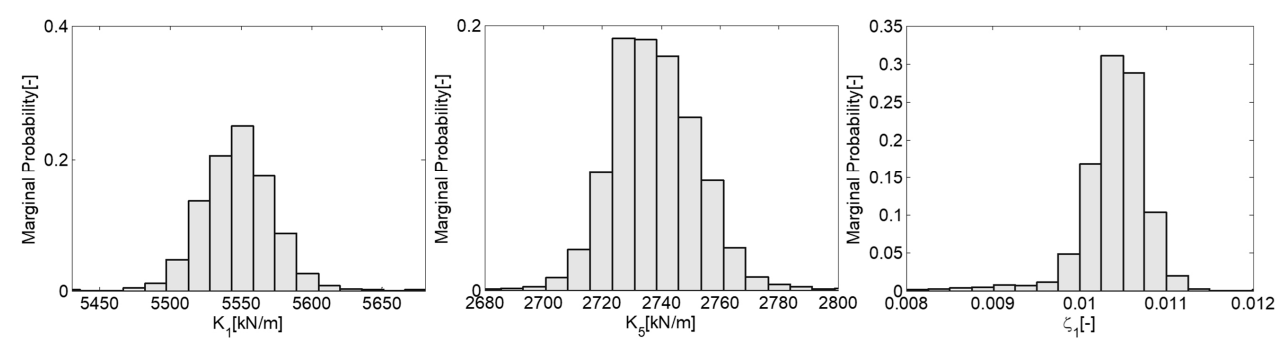

(c)

Figure 5. Marginal posterior probability distributions of the three estimation parameters $\left(k_{1}, k_{5}, \zeta_{1}\right)$ : (a) Case 1: 0\% noise + Full Outputs; (b) Case 2: 10\% noise + Full Outputs; (c) Case 3: 10\% noise + Partial Outputs. 
Table 3. Statistics of the posterior parameter distribution with the relative error (R.E.) between the posterior mean and the true value, including the standard deviation (S.D.) for each scenario.

\begin{tabular}{ccccccc}
\hline \multirow{2}{*}{ Parameter } & \multicolumn{3}{c}{ S.D. } & & \multicolumn{2}{c}{ R.E } \\
\cline { 2 - 6 } & Case1 & Case2 & Case3 & Case1 & Case2 & Case3 \\
\hline$k_{1}(\mathrm{kN} / \mathrm{m})$ & 0 & 8.81 & 25.5 & $0 \%$ & $0.16 \%$ & $0.30 \%$ \\
$k_{2}(\mathrm{kN} / \mathrm{m})$ & 0 & 4.39 & 9.55 & $0 \%$ & $0.16 \%$ & $0.35 \%$ \\
$k_{3}(\mathrm{kN} / \mathrm{m})$ & 0 & 3.6 & 8.72 & $0 \%$ & $0.13 \%$ & $0.32 \%$ \\
$k_{4}(\mathrm{kN} / \mathrm{m})$ & 0 & 4.27 & 6.56 & $0 \%$ & $0.16 \%$ & $0.24 \%$ \\
$k_{5}(\mathrm{kN} / \mathrm{m})$ & 0 & 7.49 & 1.51 & $0 \%$ & $0.28 \%$ & $0.42 \%$ \\
$k_{6}(\mathrm{kN} / \mathrm{m})$ & 0 & 4.56 & 7.3 & $0 \%$ & $0.17 \%$ & $0.28 \%$ \\
$k_{7}(\mathrm{kN} / \mathrm{m})$ & 0 & 5.44 & 8.81 & $0 \%$ & $0.20 \%$ & $0.32 \%$ \\
$k_{8}(\mathrm{kN} / \mathrm{m})$ & 0 & 6.37 & 10.3 & $0 \%$ & $0.23 \%$ & $0.38 \%$ \\
$\zeta_{1}$ & 0 & $1.64 \times 10^{-4}$ & $2.63 \times 10^{-4}$ & $0 \%$ & $1.64 \%$ & $2.63 \%$ \\
$\zeta_{2}$ & 0 & $5.8 \times 10^{-5}$ & $1.09 \times 10^{-4}$ & $0 \%$ & $0.49 \%$ & $0.76 \%$ \\
\hline
\end{tabular}

Naturally, estimates of the parameters obtained using the partial measurements are less precise. A marked decrease in accuracy can also be observed when the measurement error is increased. In partial measurements and noise polluted scenarios, Table 3 shows relative errors to be a little higher, ranging from 0.24 to $2.63 \%$, indicating a considerable measurement uncertainty (insufficient observations with errors). The damping parameters are poorly estimated, as it has small influence on the overall response generally. Hence, estimates for a damping coefficient based on measured time-domain responses may contain large uncertainties depending on the noise content in the observed data. The identified parameter set cannot be a single reliable value set due to uncertainties that persist during measurement, and therefore, should be represented with a posterior probability distribution.

Secondly, to compare the SCEM-UA methodology performance with other global optimization algorithms, such as GA, PSO, BB-BC, and SCE-UA, parameter identification for a 10-DOF structural system, used by Perry et al. [5] as a benchmark model for structural parameter estimation, is presented and structure properties are given in Table 4 . In this example, $\mathbf{M}, \mathbf{C}$, and $\mathbf{K}$ are unknown. White Gaussian noise with the RMS of the force scaled to $1000 \mathrm{~N}$ is chosen as input force loading at the 5th floor. Acceleration measurement data at floors 1, 2, 4, 6, 8, and 10 are available in the unknown mass case. Parameter estimation of the model with $10 \%$ noise scenario is investigated for the sake of comparison.

Table 4. Structure properties of the 10-DOF system.

\begin{tabular}{cc}
\hline Mass $\mathbf{( k g )}$ & \\
\hline$m_{1} \sim m_{4}$ & 6000 \\
$m_{5} \sim m_{8}$ & 4200 \\
Stiffness $(\mathrm{kN} / \mathrm{m})$ & \\
$k_{1} \sim k_{4}$ & 5000 \\
$k_{5} \sim k_{8}$ & 4000 \\
$k_{9} \sim k_{10}$ & 3000 \\
Stiffness $(\mathrm{kN} / \mathrm{m})$ & \\
First mode & 1.321 \\
Second mode & 0.505 \\
\hline
\end{tabular}

The SCEM-UA algorithm was implemented with $\mathrm{q}=30$ complexes including 20 points in each complex. The estimation parameter sets are tabulated in Table 4 . The prior uniform information is assumed. The evolution of the Gelman-Rubin factor for estimation parameters is illustrated in Figure 6. Evolutions of the samples converge rapidly as shown in Figure 7. Six thousand samples generated after convergence are used to construct the marginal posterior probability distributions for the structural model estimation parameters as illustrated in Figure 8. 


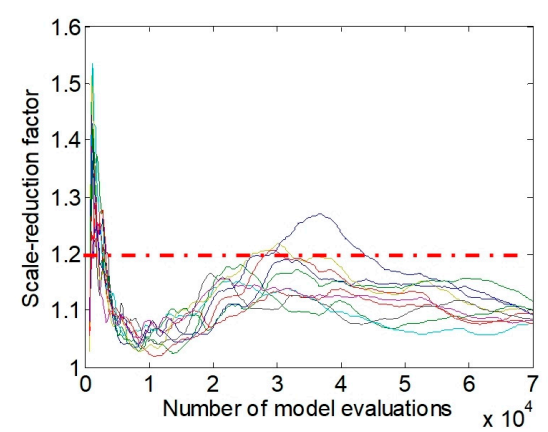

Figure 6. Evolution of the scale reduction factor for the estimation parameters.
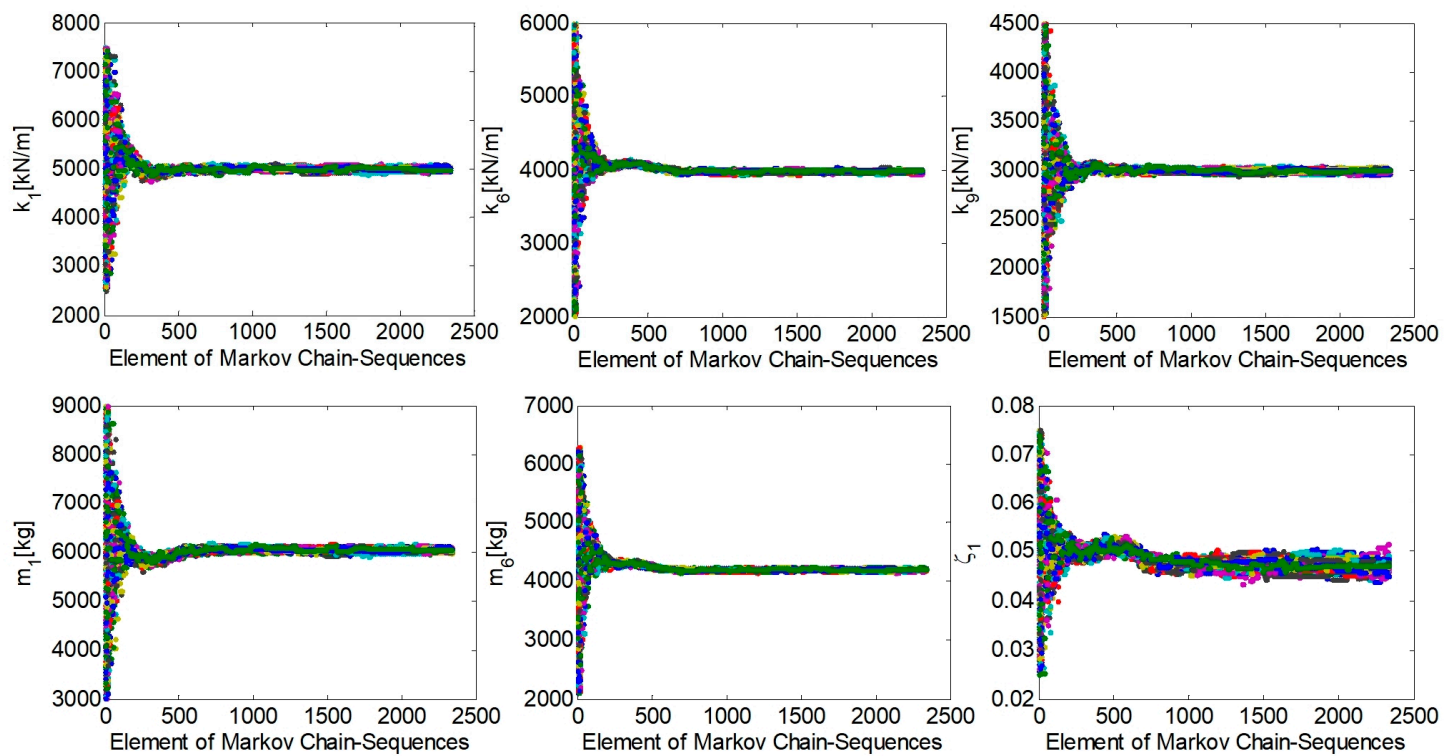

Figure 7. Generated samples for the six estimation parameters $\left(k_{1}, k_{6}, k_{9}, m_{1}, m_{6}, \zeta_{1}\right)$.
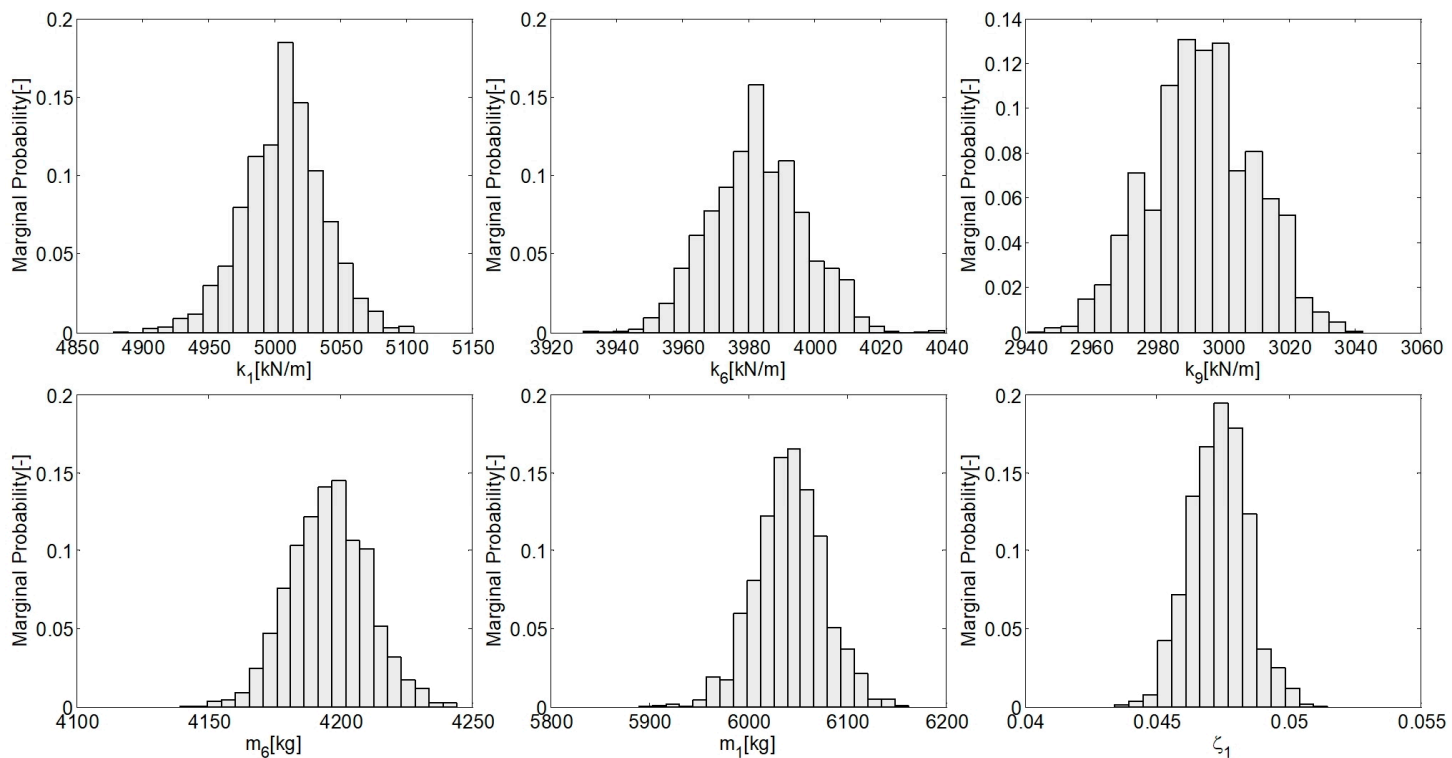

Figure 8. Marginal posterior probability distributions of the six estimation parameters $\left(k_{1}, k_{6}, k_{9}, m_{1}\right.$, $\left.m_{6}, \zeta_{1}\right)$.

Table 5 presents the final posterior moments of the generated samples after the convergence. The most likely parameter values of the structural model are listed for comparison, identified using 
the GA, PSO, BB-BC, and SCE-UA global optimization algorithms after the same evaluations $(50,000)$ for the sake of comparison. Clearly, when compared with these methods, the SCEM-UA algorithm shows a slightly better global optimization capability and can assess the uncertainties associated with the posterior distribution of the parameter estimates simultaneously within a single optimization run, which is an additional advantage of the SCEM-UA algorithm.

Table 5. Average error (avg. error) and maximum error (max. error) between the parameter value found with the lowest posterior probability and the true value.

\begin{tabular}{cccccc}
\hline Parameter & GA & PSO & BB-BC & SCE-UA & SCEM-UA \\
\hline Avg. error- $m(\%)$ & 2.98 & 2.74 & 2.61 & 1.04 & 0.68 \\
Max. error- $m(\%)$ & 6.62 & 7.11 & 4.55 & 3.17 & 1.49 \\
Avg. error- $k(\%)$ & 3.00 & 3.00 & 2.25 & 1.12 & 0.73 \\
Max. error- $k(\%)$ & 6.81 & 6.10 & 3.33 & 2.62 & 1.52 \\
Avg. error-c $(\%)$ & 8.41 & 7.96 & 2.79 & 2.94 & 2.71 \\
Max. error-c $(\%)$ & 15.29 & 10.7 & 4.72 & 6.13 & 5.40 \\
\hline
\end{tabular}

\section{Experimental Investigation}

In this section, two case studies with increasing complexity are carried out for experimental investigation to validate the feasibility of the proposed SCEM-UA algorithm. The first case is a shaking test table test of a linear system. This illustrates the capability of the algorithm to identify structural parameters and to infer the posterior target distribution. The second case study is conducted to explore the effectiveness of the algorithm for estimating the parameters of a nonlinear structure, considering the uncertainty of available information.

\subsection{Case 1: The Linear System}

A shaking table test of a 5-story steel frame model (with the model dimension shown in Figure 9) was carried out to investigate the applicability of the SCEM-UA algorithm. The $15 \times 180 \times 1060 \mathrm{~mm}$ sized columns were made of Q690 high-strength steel plates, and the $2.0 \mathrm{~m} \times 2.0 \mathrm{~m}$ sized floors were of Q345 steel plates. The structure can be simplified into a 5-story shear frame as shown in Figure 9, and the structural dynamic equation can be described as Equation (2). In this case, the structural dynamics behavior can be determined by the parameter set $\theta=\left(m_{1}, m_{2}, \ldots, m_{5}, k_{1}, k_{2}, \ldots, k_{5}, \zeta_{1}, \zeta_{2}\right)$ with the mass matrices $\mathbf{M}=(1032 \mathrm{~kg}, 1032 \mathrm{~kg}, 1032 \mathrm{~kg}, 1032 \mathrm{~kg}, 829.9 \mathrm{~kg})$ already known. The El Centro wave $(1940$, NS) was utilized in the shaking table test as the ground motion in this research, and the peak ground acceleration (PGA) was $0.15 g$ ( $g$ is the gravity acceleration). The response of the structure was measured by acceleration sensors on all floors, as shown in Figure 9.

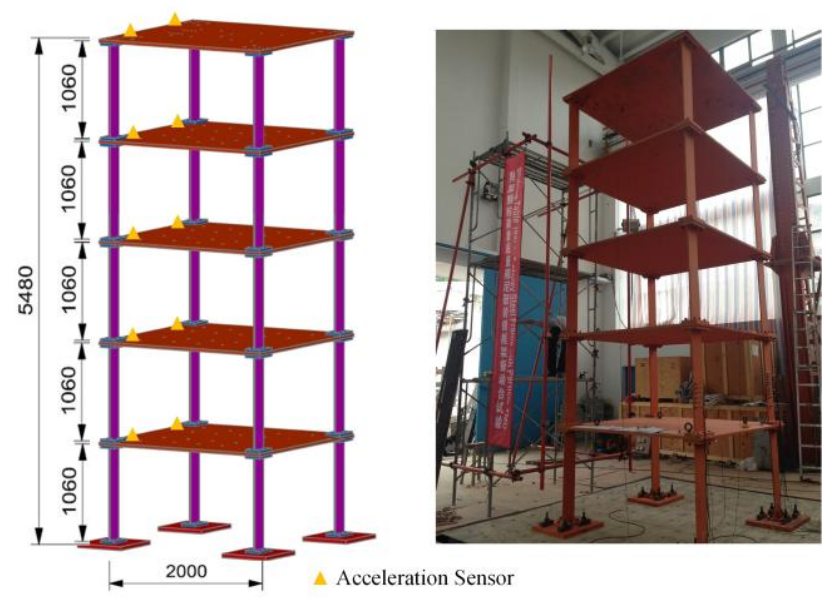

Figure 9. Schematic diagram and the test model of the 5-DOF structure system. 
The SCEM-UA algorithm was implemented $\mathrm{q}=30$ complexes including 20 points in each complex. In the inverse procedure, the upper and lower bounds on prior uniform distributions of the parameters are tabulated in Table 6 . Therefore, the feasible parameter space is defined on this rectangle.

Table 6. Bounds of the prior uniform distributions for the parameters of the 5-DOF model.

\begin{tabular}{ccc}
\hline Parameter & Lower Bound & Upper Bound \\
\hline$k_{1} \sim k_{5}(\mathrm{kN} / \mathrm{m})$ & $6.1 \times 10^{4}$ & $9.07 \times 10^{5}$ \\
$\zeta_{1}$ & $4 \times 10^{-4}$ & $6 \times 10^{-3}$ \\
$\zeta_{2}$ & $1 \times 10^{-4}$ & $1.5 \times 10^{-3}$ \\
\hline
\end{tabular}

The evolution of the Gelman-Rubin factor for each estimation parameter is illustrated in Figure 10. The parallel sequences of all parameters converged to the stationary distribution after approximately 500 runs. The generated samples rapidly converge as shown in Figure 11, which also shows the efficiency of the algorithm. Similar results are obtained for the other parameters. Six thousand samples generated after convergence are used to construct the marginal posterior probability distributions of the estimation parameters as presented in Figure 12. The marginal posterior probability distributions are quite different from the respective prior uniform distributions. The posterior can overwhelm the prior as new data are analyzed. Table 7 shows the summarizing statistics derived using those generated samples after convergence to the stationary target distribution.

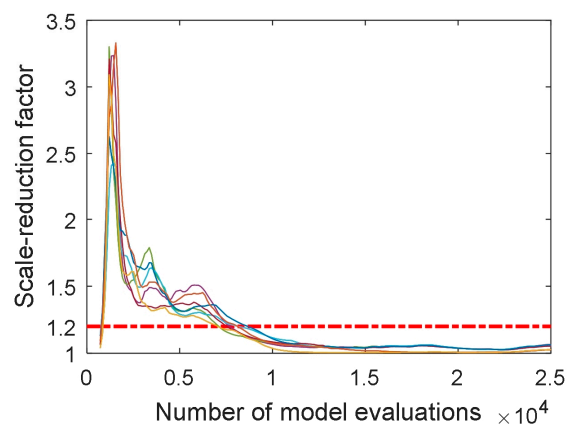

Figure 10. Evolution of the scale reduction factor for the seven estimation parameters.
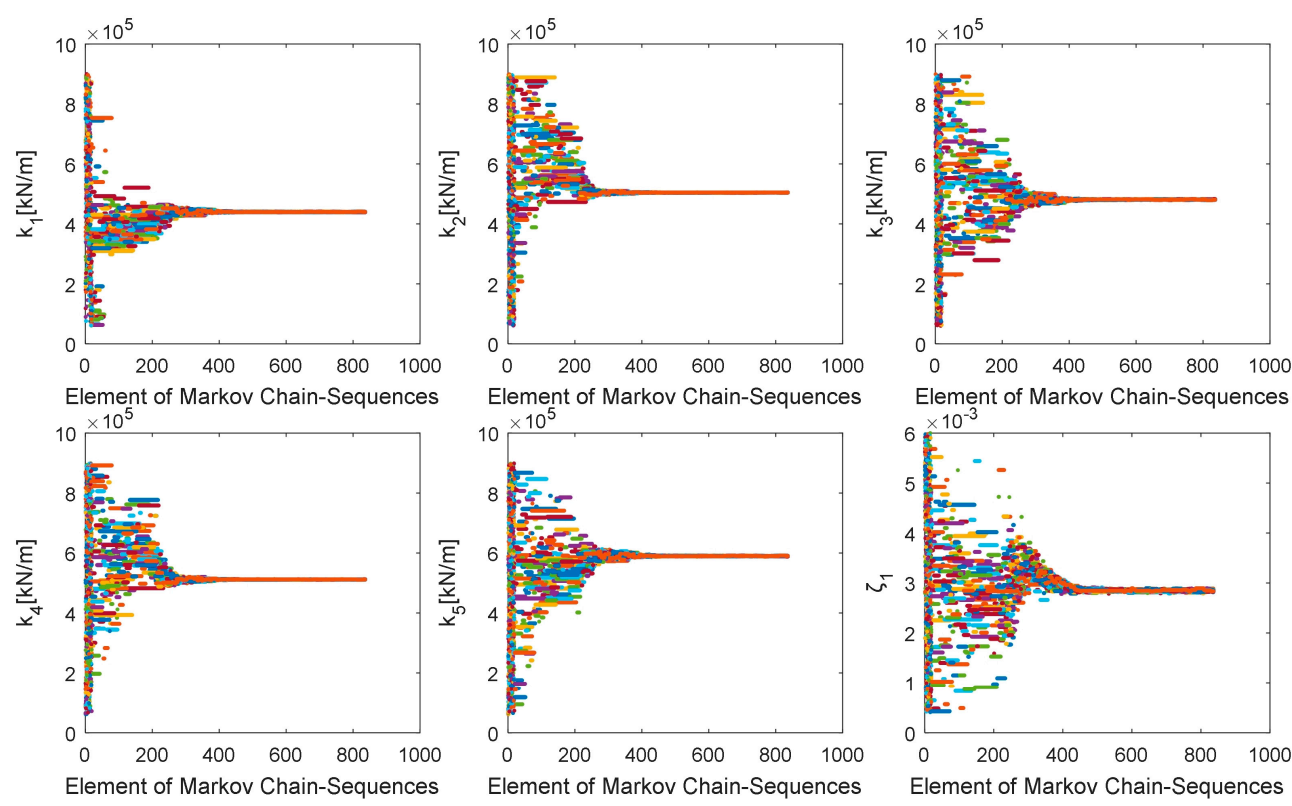

Figure 11. Generated samples for the six estimation parameters $\left(k_{1}, k_{2}, k_{3}, k_{4}, k_{5}, \zeta_{1}\right)$. 

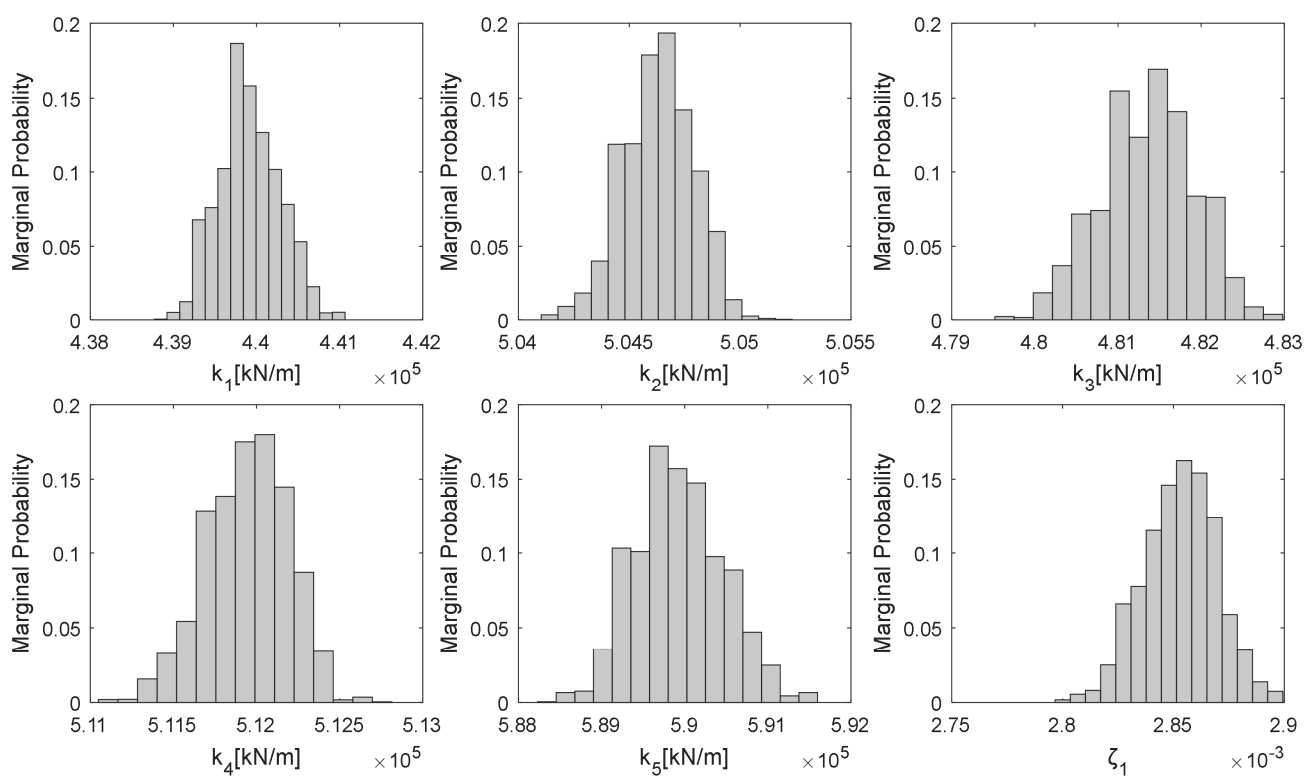

Figure 12. Marginal posterior probability distributions of the six estimation parameters $\left(k_{1}, k_{2}, k_{3}, k_{4}\right.$, $\left.k_{5}, \zeta_{1}\right)$.

Table 7. Summarizing statistics of the posterior parameter distribution mean value (M.V., standard deviation (S.D.), and relative deviation (R.E.)).

\begin{tabular}{cccc}
\hline Parameter & M.V. & S.D. & R.D. \\
\hline$k_{1}(\mathrm{kN} / \mathrm{m})$ & $4.434 \times 10^{5}$ & $2.52 \times 10^{2}$ & $0.057 \%$ \\
$k_{2}(\mathrm{kN} / \mathrm{m})$ & $5.034 \times 10^{5}$ & 96.8 & $0.019 \%$ \\
$k_{3}(\mathrm{kN} / \mathrm{m})$ & $4.755 \times 10^{5}$ & $3.65 \times 10^{2}$ & $0.077 \%$ \\
$k_{4}(\mathrm{kN} / \mathrm{m})$ & $5.107 \times 10^{5}$ & $1.73 \times 10^{2}$ & $0.034 \%$ \\
$k_{5}(\mathrm{kN} / \mathrm{m})$ & $5.980 \times 10^{5}$ & $3.75 \times 10^{2}$ & $0.063 \%$ \\
$\zeta_{1}$ & $2.5 \times 10^{-3}$ & $2.58 \times 10^{-6}$ & $0.10 \%$ \\
$\zeta_{2}$ & $8.59 \times 10^{-4}$ & $1.18 \times 10^{-6}$ & $0.14 \%$ \\
\hline
\end{tabular}

To demonstrate the reliability of the identified values, the identified results are used to re-simulate the structural response by the given excitation. The comparison results of the acceleration histories at the $3 \mathrm{rd}$ and 5 th floor between the experiment data and the best simulation results, which have the largest likelihood value based on the principle of Bayesian statistics, are shown in Figure 13. After convergence was accomplished, the last $10 \%$ of the samples that adequately fit the model responses onto observations were exerted to recalculate model outputs. Figure 14 demonstrates the $95 \%$ acceleration histories prediction uncertainty depicted by computing $2.5 \%$ and $97.5 \%$ percentiles associated with the posterior distribution of the parameter estimates. In this linear system, the sources of uncertainty are considered as the measurement uncertainty and model uncertainty. The simplification, inadequacy, and ambiguity in description of realistic structural system through mathematical equation leads to model structural uncertainty. The results show the SCEM-UA algorithm has feasibility with good accuracy and is able to estimate the uncertainties associated with the posterior distribution of the unknown parameters simultaneously within a single optimization run. 


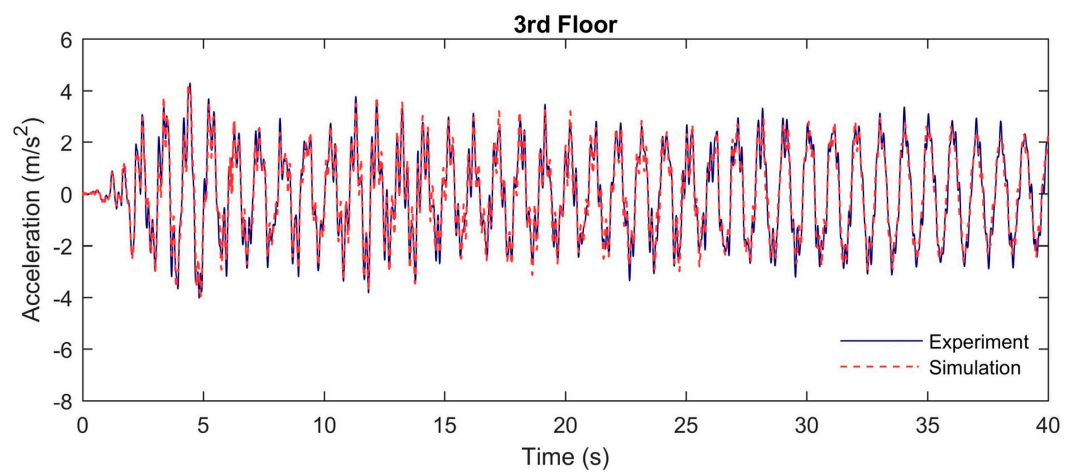

(a)

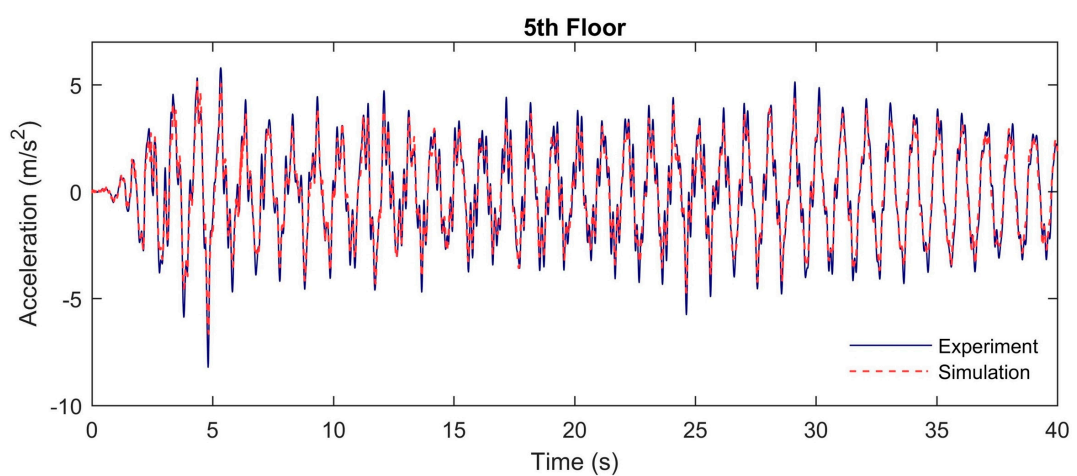

(b)

Figure 13. Experimental and simulation acceleration time histories in the linear structural system: (a) At the 3rd floor; (b) At the 5th floor.

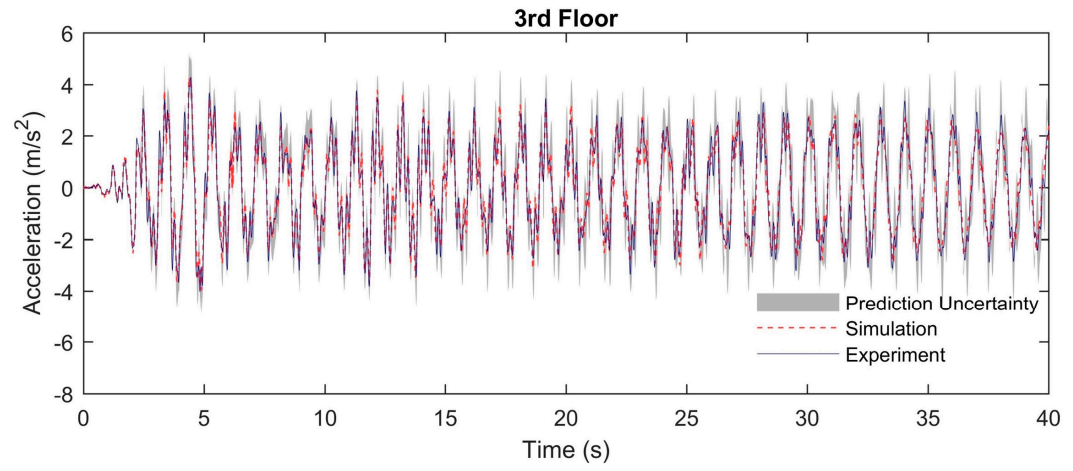

(a)

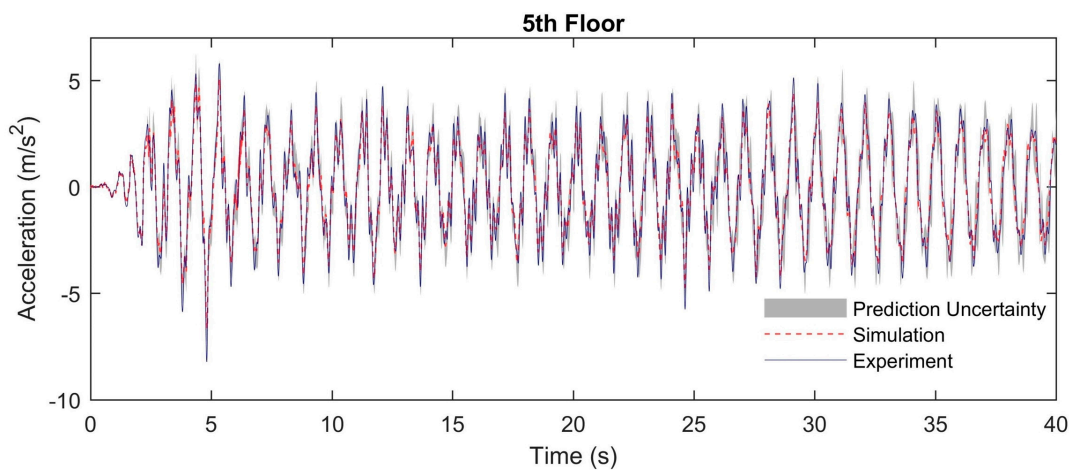

(b)

Figure 14. Experimental and simulation acceleration time histories in the linear structural system: (a) At the 3rd floor; (b) At the 5th floor. 


\subsection{Case 2: The Nonlinear System}

The results of this case study thus far indicate that the SCEM-UA algorithm is capable of parameter identification with incomplete measurements of the structural response and existing noise. In this section, the algorithm is applied to a nonlinear structural system, which is a realistic 5-story steel frame with a particle tuned mass damper (PTMD). The PTMD, combining the particle damping technology and TMDs, possess multiple damping mechanisms [34]. Considering the calculation efficiency, the simplified analytical method proposed by Lu et al. [35] is selected to simulate the vibration reduction effects of the PTMD. The particles in the container are equivalent to a single particle and the schematic diagram of the primary structure with the PTMD as illustrated by Figure 15. The suspended container is considered as the $(\mathrm{n}+1)$ th DOF with an external force $F_{\mathrm{p}}$ caused by the collisions between the container and the particle. Therefore, the governing equation of the primary structure can be rewritten as

$$
\begin{gathered}
\left\{\begin{array}{c}
\mathbf{M} \ddot{\mathbf{x}}(t)+\mathbf{C} \dot{\mathbf{x}}(t)+\mathbf{K} \mathbf{x}(t)=\mathbf{E} \ddot{x} g+\phi\left(c_{\mathrm{c}} \dot{y}_{1}+k_{\mathrm{c}} y_{1}\right) \\
m_{\mathrm{c}} \ddot{x}_{\mathrm{c}}+c_{\mathrm{c}} \ddot{y}_{1}+k_{\mathrm{c}} y_{1}-c_{\mathrm{p}} H\left(y_{2}, \dot{y}_{2}\right)-k_{\mathrm{p}} G\left(y_{2}\right)=0 \\
m_{\mathrm{p}} \ddot{x}_{\mathrm{p}}+c_{\mathrm{p}} H\left(y_{2}, \dot{y}_{2}\right)+k_{\mathrm{p}} G\left(y_{2}\right)=0
\end{array}\right. \\
\mathbf{E}=\left[\begin{array}{lll}
-m_{1}-m_{2} & \ldots & -m_{\mathrm{n}}
\end{array}\right]^{T}, \\
\phi=\left[\begin{array}{llll}
0 & 0 & \ldots & 0
\end{array}\right]^{T},
\end{gathered}
$$

where $\phi$ is the location vector of the control force, $y_{1}=x_{\mathrm{c}}-x_{5}, y_{2}=x_{\mathrm{p}}-x_{\mathrm{c}}$, and the container and the simplified particle are represented by the subscripts $\mathrm{c}$ and $\mathrm{p}$, respectively. $G\left(y_{2}\right)$ and $\mathrm{H}\left(y_{2}, \dot{y}_{2}\right)$ are nonlinear functions related to the clearance $d$ of the equivalent single-particle damper, which can be obtained based on the equivalent principle. In addition, $k_{\mathrm{c}}=\omega_{\mathrm{c}}^{2} m_{\mathrm{c}}$ and $c_{\mathrm{c}}=2 m_{\mathrm{c}} \xi_{\mathrm{c}} \omega_{\mathrm{c}}$ represent the interactions between the primary structure and the container. $k_{\mathrm{p}}=\omega_{\mathrm{p}}^{2} m_{\mathrm{p}}$ and $c_{\mathrm{p}}=2 m_{\mathrm{p}} \xi_{\mathrm{p}} \omega_{\mathrm{p}}$ represent collisions between the container and the particles. More details about the equivalent principle and the simplified analytical method are given by Lu et al. [35].
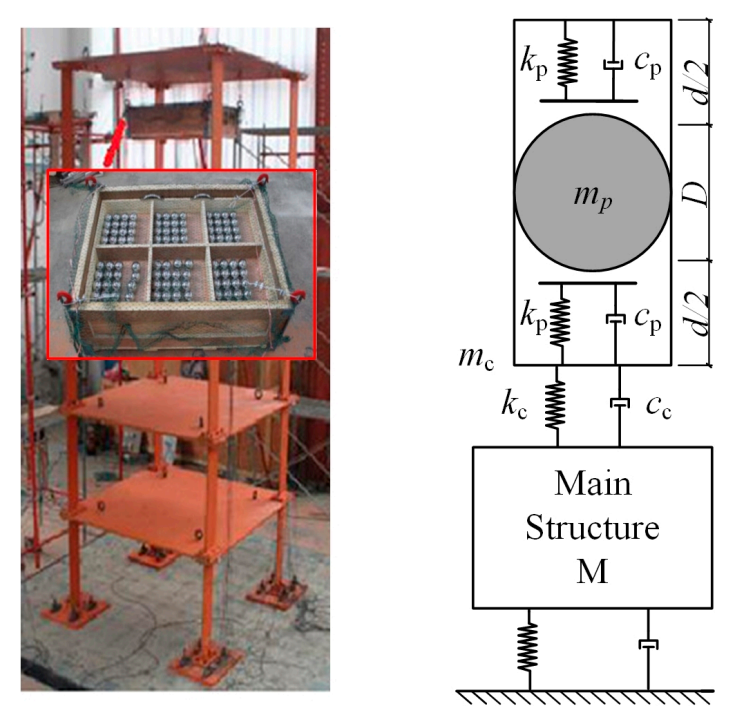

Figure 15. The test model and a simplified schematic of the system.

The PTMD with a $2.26 \%$ total auxiliary mass ratio to the primary system is suspended on top of the primary steel frame by four steel strands. The dynamic equation of the structure's system is discussed in Sections 3 and 4.1. The mass matrix of the steel frame $\mathbf{M}=(1032 \mathrm{~kg}, 1032 \mathrm{~kg}, 1032 \mathrm{~kg}, 1032 \mathrm{~kg}$, $829.9 \mathrm{~kg}$ ), and the total masses of the container and the particles, $m_{\mathrm{c}}=39.345 \mathrm{~kg}$ and $m_{\mathrm{p}}=7.96 \mathrm{~kg}$, are already known. In addition, based on the fundamental theorem of the structural dynamics, the stiffness and the damping coefficient can be determined by the circular frequency and the damping 
ratio, respectively. Therefore, the parameters of the structure system, which are unable to be measured can be described by the parameter set

$$
\theta=\left(k_{1}, k_{2}, \ldots, k_{5}, \zeta_{1}, \zeta_{2}, \omega_{\mathrm{c}}, \zeta_{\mathrm{c}}, \omega_{\mathrm{p}}, \zeta_{\mathrm{p}}\right)
$$

In this study, the structure system response excited by the one-dimensional Shanghai artificial wave (SHW2, 1996) ground motion is selected as an example and the peak value of the acceleration is $0.05 \mathrm{~g}$. Acceleration measurements at floors 1,3, 5 are available in this case. The SCEM-UA algorithm was implemented with $\mathrm{q}=20$ complexes including 20 points in each complex. The prior information of estimation parameters consists of realistic lower and upper bounds on each of the parameters with a uniform prior distribution as tabulated in Table 8.

Table 8. Bounds of the prior uniform distributions for the parameters of the nonlinear structural system.

\begin{tabular}{ccc}
\hline Parameter & Lower Bound & Upper Bound \\
\hline$k_{1} \sim k_{5}(\mathrm{kN} / \mathrm{m})$ & $1 \times 10^{5}$ & $1 \times 10^{6}$ \\
$\zeta_{1}$ & $1.5 \times 10^{-5}$ & $7.5 \times 10^{-3}$ \\
$\zeta_{2}$ & $5 \times 10^{-6}$ & $2.5 \times 10^{-3}$ \\
$\omega_{\mathrm{c}}(\mathrm{rad} / \mathrm{s})$ & $5 \times 10^{-2}$ & 25 \\
$\omega_{\mathrm{p}}(\mathrm{rad} / \mathrm{s})$ & 1 & $5 \times 10^{2}$ \\
$\zeta_{\mathrm{c}}$ & $1 \times 10^{-3}$ & $5 \times 10^{-1}$ \\
$\zeta_{\mathrm{p}}$ & $2 \times 10^{-3}$ & 1.0 \\
\hline
\end{tabular}

The evolution of the Gelman-Rubin scale reduction factor for estimation parameters using the SCEM-UA algorithm is illustrated in Figure 16, indicating that the parallel sequences converged to the stationary distribution after approximately 400 iterations. The evolution of the generated samples converges rapidly as presented in Figure 17. Similar results were obtained for the other parameters. Figure 18 presents the marginal posterior probability distributions of the estimated parameters constructed after convergence. Note that none of the parameters in Figure 18 correspond to the respective prior uniform distributions. Table 9 presents summarizing statistics of the posterior parameter distributions. In general, the width of posterior distribution in a nonlinear system is relatively larger than in a linear system.

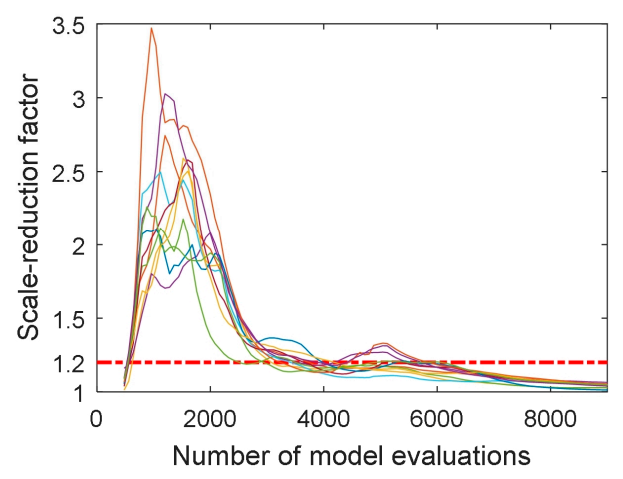

Figure 16. Evolution of the scale reduction factor for the 11 estimation parameters. 

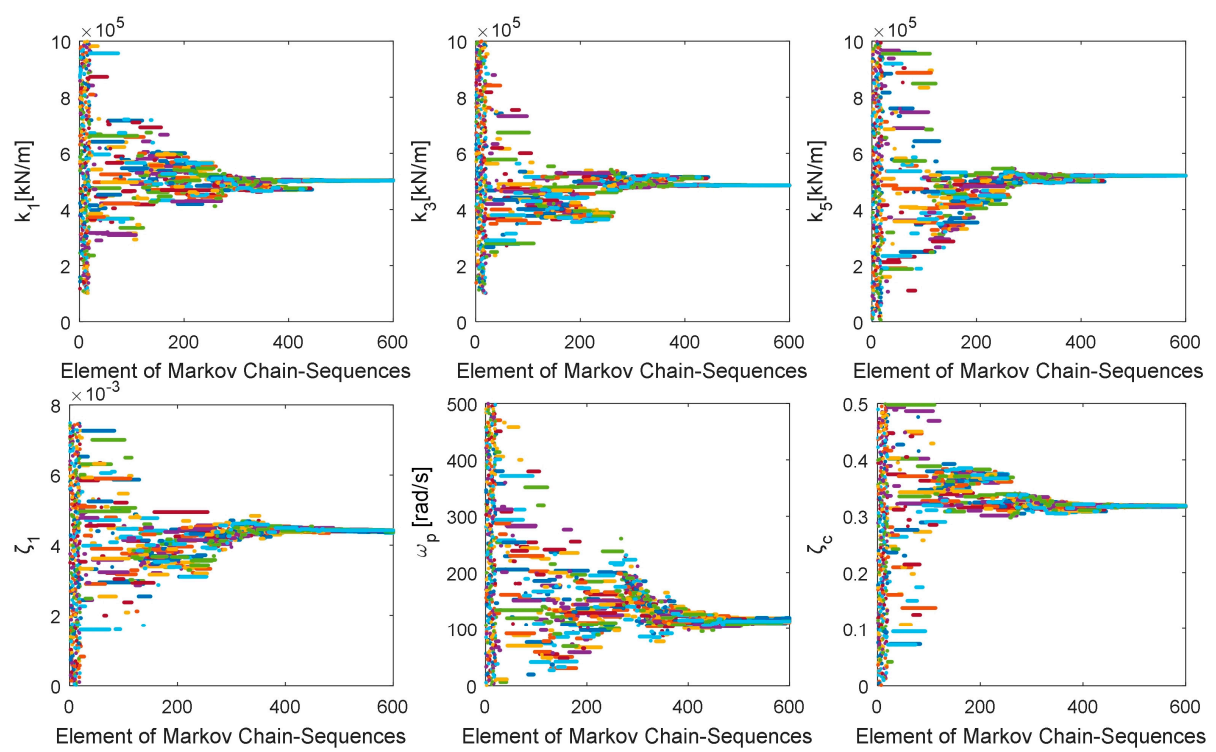

Figure 17. Generated samples for the six estimation parameters $\left(k_{1}, k_{3}, k_{5}, \zeta_{1}, \omega_{\mathrm{p}}, \zeta_{\mathrm{c}}\right)$.
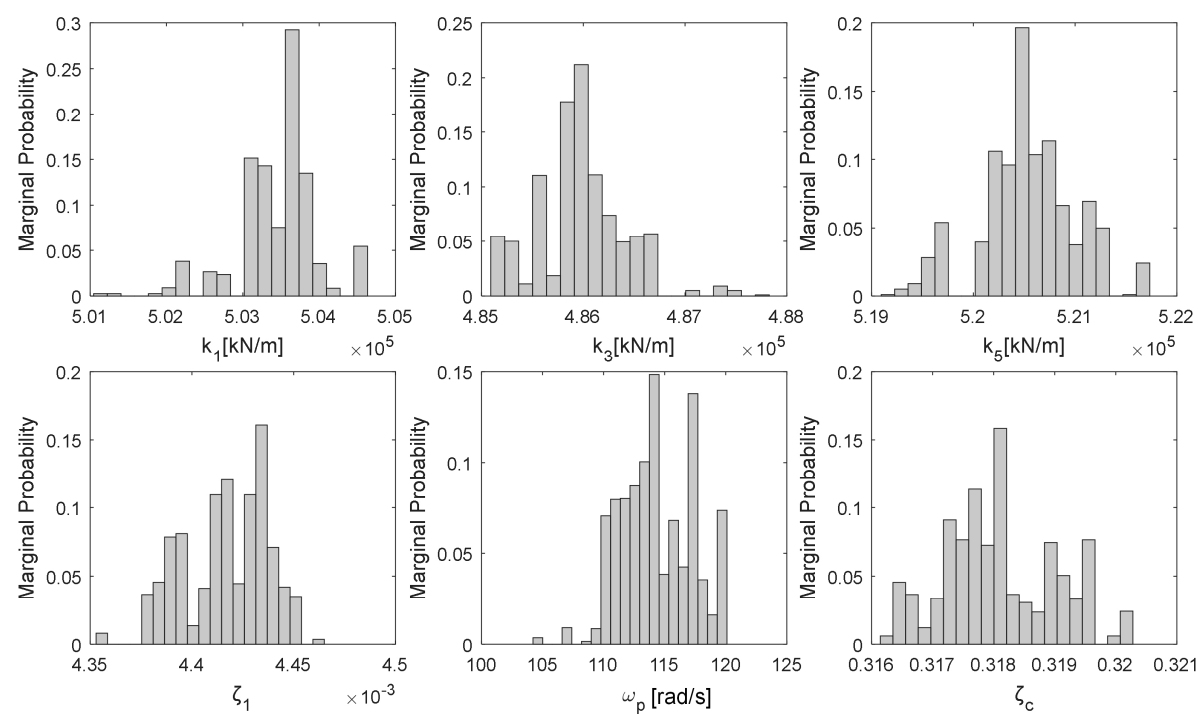

Figure 18. Marginal posterior probability distributions of the six estimation parameters $\left(k_{1}, k_{3}, k_{5}, \zeta_{1}\right.$, $\left.\omega_{\mathrm{p}}, \zeta_{\mathrm{c}}\right)$.

Table 9. Summarizing statistics of the posterior parameter distribution (Mean Value (M.V.), Standard Deviation (S.D.), and Relative Deviation (R.E.)).

\begin{tabular}{cccc}
\hline Parameter & M.V. & S.D. & R.D. \\
\hline $\mathrm{k} 1(\mathrm{kN} / \mathrm{m})$ & $5.011 \times 10^{5}$ & $7.73 \times 10^{3}$ & $1.54 \%$ \\
$\mathrm{k} 2(\mathrm{kN} / \mathrm{m})$ & $5.619 \times 10^{5}$ & $4.45 \times 10^{3}$ & $0.79 \%$ \\
$\mathrm{k} 3(\mathrm{kN} / \mathrm{m})$ & $4.879 \times 10^{5}$ & $6.98 \times 10^{3}$ & $1.43 \%$ \\
$\mathrm{k} 4(\mathrm{kN} / \mathrm{m})$ & $4.721 \times 10^{5}$ & $5.61 \times 10^{3}$ & $1.19 \%$ \\
$\mathrm{k} 5(\mathrm{kN} / \mathrm{m})$ & $5.190 \times 10^{5}$ & $4.93 \times 10^{3}$ & $0.95 \%$ \\
$\zeta 1$ & $4.423 \times 10^{-3}$ & $3.11 \times 10^{-5}$ & $0.70 \%$ \\
$\zeta_{2}$ & $6.601 \times 10^{-4}$ & $3.79 \times 10^{-6}$ & $0.57 \%$ \\
$\omega_{\mathrm{c}}(\mathrm{rad} \cdot \mathrm{s})$ & $1.584 \times 10^{2}$ & $2.3 \times 10^{-1}$ & $1.45 \%$ \\
$\omega_{\mathrm{p}}(\mathrm{rad} \cdot \mathrm{s})$ & $1.14 \times 10^{2}$ & 4.94 & $4.33 \%$ \\
$\zeta_{\mathrm{c}}$ & $3.175 \times 10^{-1}$ & $2.38 \times 10^{-3}$ & $0.75 \%$ \\
$\zeta_{\mathrm{p}}$ & $2.621 \times 10^{-1}$ & $1 \times 10^{-2}$ & $3.81 \%$ \\
\hline
\end{tabular}


To demonstrate the reliability of the identified values, the identified model parameters are input to operate the simulation of the structural response to the given excitation. The simulation results of the acceleration histories at the 3rd and 5th floor in this study are shown in Figure 19 as dashed curves, whereas the solid curves are the corresponding experimental acceleration histories for comparison. The PTMD performs nonlinearly under seismic excitation, but the results of the proposed identification algorithm generally matched. After the convergence was accomplished, the last $10 \%$ of the samples were employed to recalculate model outputs, and then by computing $2.5 \%$ and $97.5 \%$ percentiles, the authors obtained a $95 \%$ confidence interval. Figure 20 demonstrates the $95 \%$ acceleration histories prediction uncertainty associated with the posterior distribution of the parameter estimates.

The predicted motion coincides with reasonably accurate estimates of the structural response showing the feasibility of the SCEM-UA algorithm for structural parameter identification and the ability to simultaneously identify the parameters of the primary structure and the PTMD. The prediction uncertainty bounds include almost all observed values during the calibration period. Note that compared with the linear structural system (Figure 14), the prediction uncertainty ranges are quite large, indicating considerable uncertainty given the current model structure. The results of structural parameter identification contain uncertainties caused by both the existing noise in the observed data due to the limitation of measurement accuracy and mechanical model simplification. The simplified model is not entirely consistent with the actual physical model, which the algorithm cannot consider. Further, the acceleration histories prediction uncertainty solely corresponding to the posterior distribution of the parameter estimates displays a bias in a certain time period, indicating that the further improvement of the computational model is necessary.

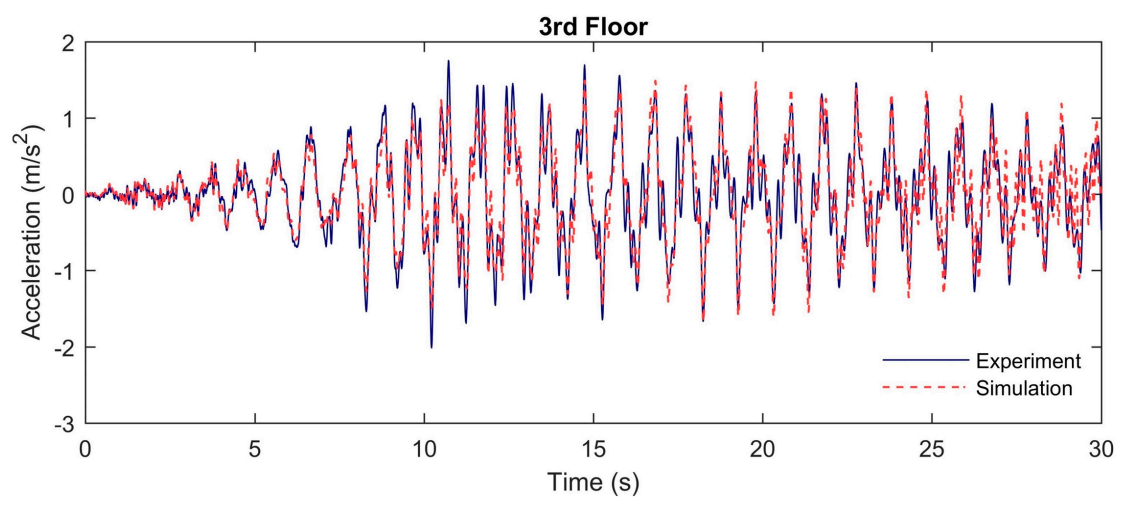

(a)

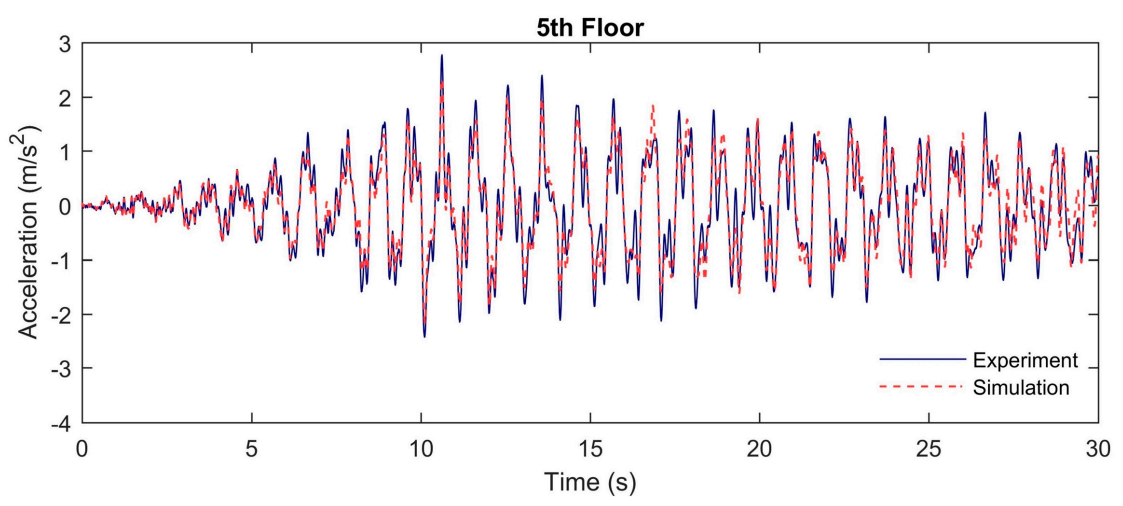

(b)

Figure 19. Experimental and simulation acceleration time histories in the nonlinear structural system: (a) At the 3rd floor; (b) At the 5th floor. 


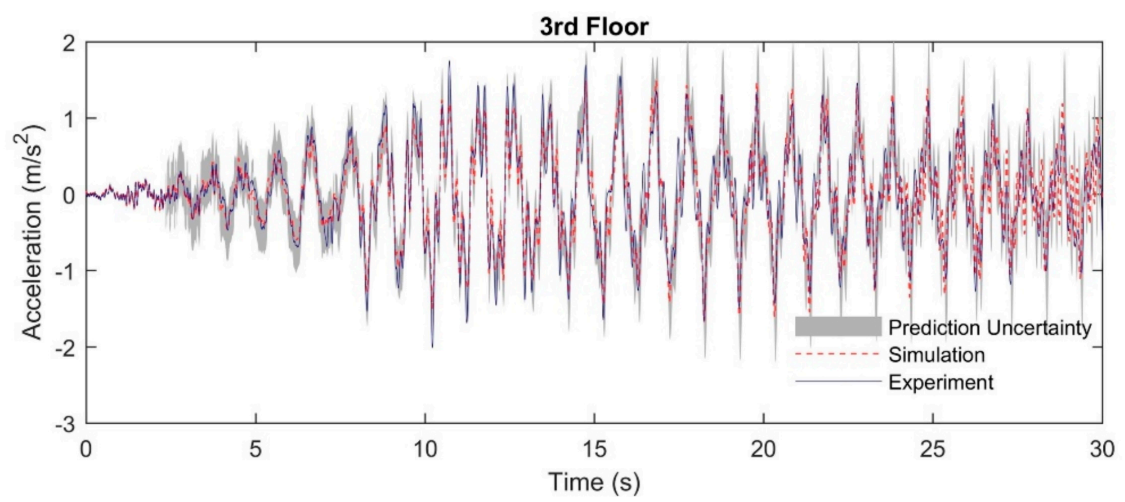

(a)

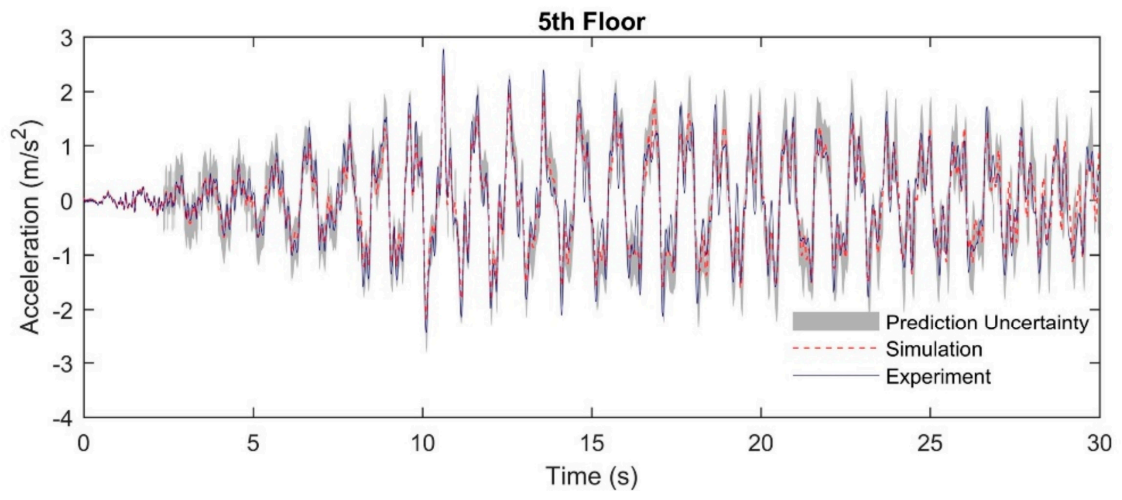

(b)

Figure 20. Prediction uncertainty ranges of acceleration histories in the nonlinear structural system:

(a) At the 3rd floor; (b) At the 5th floor.

\section{Conclusions}

In this research, the authors developed a structural optimal parameter and uncertainty estimation method using the SCEM-UA algorithm, which mainly focused on uncertain parameter distributions. Parameter identification has been successfully investigated based on numerical simulations and laboratory experiments. The main conclusions of this research are:

1. The SCEM-UA algorithm can effectively explore the feasible parameter space to locate the high probability density region and converge to the stationary posterior parameter distributions for structural systems under seismic excitation.

2. The sampling of the SCEM-UA algorithm requires no prior distribution, which can better estimate the posterior distribution of complex structural model parameters with very little prior information.

3. The SCEM-UA method shows slightly better global optimization capability compared with the other global optimization algorithms, and the SCEM-UA algorithm focused on model uncertainty analysis especially. The SCEM-UA algorithm not only searches the posterior distribution of model parameters but also deduces the fitting of a model's calculated and observed values under a certain confidence interval. Moreover, many parameter sets close to the global optimum behave with quite similar performances in reproducing the observed data.

4. Parameter uncertainties, including measurement uncertainty and model uncertainty, do exist in the structural system as posterior probability density distributions, and the results obtained showed wide demand prediction uncertainty bounds. Moreover, the prediction uncertainty bounds are wider in the nonlinear structural systems than in the linear structural systems due to the nonlinear model simplification. 
However, this research only considered the uncertain source of insufficient observations with errors. In the future, the SCEM-UA approach presented here will be employed to assess the uncertainty in parameter estimates of real structural models including modeling, observation, and excitation uncertainties. In addition, if the independent errors assumption does not exist, the estimation of the parameter posterior distributions will be affected. Future studies will also address this issue.

Author Contributions: Conceptualization and Methodology, H.T.; Investigation and Validation, X.G.; Writing-Original Draft Preparation, H.T., and X.G.; Writing_Review and Editing, H.T., L.X. and S.X.

Funding: This study was supported by the Ministry of Science and Technology of China (Grant No. SLDRCE19-B-02), the National Key Research and Development Program (2016YFC0701800, 2017YFC0703607) and the Natural Science Foundation of Shanghai (Grant No. 17ZR1431900).

Conflicts of Interest: The authors declare no conflict of interest.

\section{References}

1. Mukhopadhyay, S.; Lus, H.; Betti, R. Structural identification with incomplete instrumentation and global identifiability requirements under base excitation. Struct. Control Health Monit. 2015, 22, 1024-1047. [CrossRef]

2. Xue, S.; Wen, B.; Huang, R.; Huang, L.; Sato, T.; Xie, L.; Tang, H.; Wan, C. Parameter identification for structural health monitoring based on Monte Carlo method and likelihood estimate. Int. J. Distrib. Sens. Netw. 2018, 14, 1550147718786888. [CrossRef]

3. Zecchin, A.C.; White, L.B.; Lambert, M.F.; Simpson, A.R. Parameter identification of fluid line networks by frequency-domain maximum likelihood estimation. Mech. Syst. Signal Process. 2013, 37, 370-387. [CrossRef]

4. Molina, D.; Latorre, A.; Herrera, F. An insight into bio-inspired and evolutionary algorithms for global optimization: Review, analysis, and lessons learnt over a decade of competitions. Cogn. Comput. 2018, 10, 517-544. [CrossRef]

5. Perry, M.J.; Koh, C.G.; Choo, Y.S. Modified genetic algorithm strategy for structural identification. Comput. Struct. 2006, 84, 529-540. [CrossRef]

6. Mokshin, A.V.; Mokshin, V.V.; Sharnin, L.M. Adaptive genetic algorithms used to analyze behavior of complex system. Commun. Nonlinear Sci. Numer. Simul. 2019, 71, 174-186. [CrossRef]

7. Tang, H.; Xue, S.; Fan, C. Differential evolution strategy for structural system identification. Comput. Struct. 2008, 86, 2004-2012. [CrossRef]

8. Opara, K.R.; Arabas, J. Differential Evolution: A survey of theoretical analyses. Swarm Evol. Comput. 2019, 44, 546-558. [CrossRef]

9. Eberhart, R.C.; Shi, Y. Particle Swarm Optimization: Developments, Applications and Resources. In Proceedings of the Congress on Evolutionary Computation, Seoul, Korea, 27-30 May 2001; pp. 81-86.

10. Lin, Q.; Liu, S.; Zhu, Q.; Tang, C.; Song, R.; Chen, J.; Coello, C.A.C.; Wong, K.C.; Zhang, J. Particle swarm optimization with a balanceable fitness estimation for many-objective optimization problems. IEEE Trans. Evol. Comput. 2016, 22, 32-46. [CrossRef]

11. Tang, H.; Jin, Z.; Xue, S.; Xie, L. Big Bang-Big Crunch optimization for parameter estimation in structural systems. Mech. Syst. Signal Process. 2010, 24, 2888-2897. [CrossRef]

12. Franco, G.; Betti, R.; Luş, H. Identification of structural systems using an evolutionary strategy. J. Eng. Mech. 2004, 130, 1125-1139. [CrossRef]

13. Levin, R.I.; Lieven, N.A.J. Dynamic finite element model updating using simulated annealing and genetic algorithms. Mech. Syst. Signal Process. 1998, 12, 91-120. [CrossRef]

14. Duan, Q.; Sorooshian, S.; Gupta, V. Effective and efficient gobal optimization for conceptual rainfall-runoff models. Water Resour. Res. 1992, 28, 1015-1031. [CrossRef]

15. Pourasghar, B.; Ahandani, M.A.; Kharrati, H. Parameter identification of engineering problems using a differential shuffled complex evolution. Artif. Intell. Rev. 2019, 1-34. [CrossRef]

16. Emery, A.F. Estimating deterministic parameters by Bayesian inference with emphasis on estimating the uncertainty of the parameters. Inverse Probl. Sci. Eng. 2009, 17, 263-274. [CrossRef] 
17. Vrugt, J.A.; Gupta, H.V.; Bouten, W.; Sorooshian, S. A Shuffled Complex Evolution Metropolis algorithm optimization and uncertainty assessment of hydrologic models parameters. Water Resour. Res. 2003, 39, 113-117. [CrossRef]

18. Beck, J.L.; Au, S.-K. Bayesian updating of structural models and reliability using Markov chain Monte Carlo simulation. J. Eng. Mech. 2002, 128, 380-391. [CrossRef]

19. Lam, H.; Hu, J.; Zhang, F.; Ni, Y. Markov chain Monte Carlo-based Bayesian model updating of a sailboat-shaped building using a parallel technique. Eng. Struct. 2019, 193, 12-27. [CrossRef]

20. Nevitt, J.; Hancock, G.R. Performance of bootstrapping approaches to model test statistics and parameter standard error estimation in structural equation modeling. Struct. Equ. Model. A Multidiscip. J. 2001, 8, 353-377. [CrossRef]

21. Li, S.J.; Suzuki, Y.; Noori, M. Identification of hysteretic systems with slip using bootstrap filter. Mech. Syst. Signal Process. 2004, 18, 781-795. [CrossRef]

22. Cara, J. Modal identification of structures from input/output data using the expectation-maximization algorithm and uncertainty quantification by mean of the bootstrap. Struct. Control Health Monit. 2019, 26, e2272. [CrossRef]

23. Glaser, R.E.; Lee, C.L.; Nitao, J.J.; Hanley, W.G. A Markov Chain Monte Carlo Based Method for System Identification; Lawrence Livermore National Lab: Livermore, CA, USA, 2002.

24. Ninness, B.; Henriksen, S. Bayesian system identification via Markov chain Monte Carlo techniques. Automatica 2010, 46, 40-51. [CrossRef]

25. Lam, H.F.; Yang, J.H.; Hu, Q.; Ng, C.T. Railway ballast damage detection by Markov chain Monte Carlo-based Bayesian method. Struct. Health Monit. 2018, 17, 706-724. [CrossRef]

26. Price, W. Global optimization algorithms for a CAD workstation. J. Optim. Theory Appl. 1987, 55, $133-146$. [CrossRef]

27. Holland, J.H. Adaptation in Natural and Artificial Systems: An Introductory Analysis with Applications to Biology, Control, and Artificial Intelligence; MIT Press: Cambridge, MA, USA, 1992.

28. Metropolis, N.; Rosenbluth, A.W.; Rosenbluth, M.N.; Teller, A.H.; Teller, E. Equation of state calculations by fast computing machines. J. Chem. Phys. 1953, 21, 1087-1092. [CrossRef]

29. Feyen, L.; Vrugt, J.A.; Nualláin, B.Ó.; Knijff, J.V.D.; Roo, A.D. Parameter optimisation and uncertainty assessment for large-scale streamflow simulation with the LISFLOOD model. J. Hydrol. 2007, 332, $276-289$. [CrossRef]

30. Her, Y.; Heatwole, C. Comparing impacts of parameter and spatial data uncertainty for a grid-based distributed watershed model. J. Hydroinform. 2016, 18, 961-974. [CrossRef]

31. Krishnan, N.; Raj, C.; Chaubey, I.; Sudheer, K. Parameter estimation of SWAT and quantification of consequent confidence bands of model simulations. Environ. Earth Sci. 2018, 77, 470. [CrossRef]

32. Vrugt, J.A.; Gupta, H.V.; Dekker, S.C.; Sorooshian, S.; Wagener, T.; Bouten, W. Application of stochastic parameter optimization to the Sacramento Soil Moisture Accounting model. J. Hydrol. 2006, 325, $288-307$. [CrossRef]

33. Gelman, A.; Rubin, D.B. Inference from iterative simulation using multiple sequences. Stat. Sci. 1992, 7 , 457-472. [CrossRef]

34. Zheng, L.; Wang, Z.; Masri, S.F.; Lu, X. Particle impact dampers: Past, present, and future. Struct. Control Health Monit. 2017, 25, e2058.

35. Zheng, L.; Chen, X.; Zhang, D.; Dai, K. Experimental and analytical study on the performance of particle tuned mass dampers under seismic excitation. Earthq. Eng. Struct. Dyn. 2017, 46, 697-714.

(C) 2019 by the authors. Licensee MDPI, Basel, Switzerland. This article is an open access article distributed under the terms and conditions of the Creative Commons Attribution (CC BY) license (http://creativecommons.org/licenses/by/4.0/). 